\title{
Adsorption of Self-Assembled Rigid Rods on Two-Dimensional Lattices
}

\author{
L. G. López and A. J. Ramirez-Pastor* \\ Departamento de Física, Instituto de Física Aplicada, Universidad Nacional de San \\ Luis-CONICET, Ejército de los Andes 950, D5700BWS San Luis, Argentina \\ E-mail: Iglopez@unsl.edu.ar,antorami@unsl.edu.ar
}

\begin{abstract}
Monte Carlo (MC) simulations have been carried out to study the adsorption on square and triangular lattices of particles with two bonding sites that, by decreasing temperature or increasing density, polymerize reversibly into chains with a discrete number of allowed directions and, at the same time, undergo a continuous isotropic-nematic (IN) transition. The process has been monitored by following the behavior of the adsorption isotherms (chemical potential $\mu$ as a function of the surface coverage $\theta$ ) for different values of lateral interaction energy/temperature. The numerical data were compared with mean-field analytical predictions and exact functions for noninteracting and 1D systems. The obtained results revealed the existence of three adsorption regimes in temperature. (1) At high temperatures, above the critical one characterizing the IN transition at full coverage $T_{c}(\theta=1)$, the particles are distributed at random on the surface and the adlayer behaves as a noninteracting 2D system. (2) At very low temperatures, the asymmetric monomers adsorb forming chains over almost the entire range of coverage, and the adsorption process behaves as a 1D problem. (3) In the intermediate regime, the system exhibits a mixed regime and the filling of the lattice proceeds according to two
\end{abstract}

\footnotetext{
*To whom correspondence should be addressed
} 
different processes. In the first stage, the monomers adsorb isotropically on the lattice until the IN transition occurs in the system and, from this point, particles adsorb forming chains so that the adlayer behaves as a 1D fluid. The two adsorption processes are present in the adsorption isotherms, and a marked singularity can be observed that separates both regimes. Thus, the adsorption isotherms appear as sensitive quantities with respect to the IN phase transition, allowing us (i) to reproduce the phase diagram of the system for square lattices and (ii) to obtain an accurate determination of the phase diagram for triangular lattices. 


\section{Introduction}

The adsorption of gases on solid surfaces is a topic of fundamental interest for various applications. ${ }^{1-3}$ From the theoretical point of view, the process can be described in terms of the latticegas theory $1-8$ or its generalizations, including lateral interactions between adsorbed particles, surface heterogeneity, multilayer adsorption, etc. -17 In all cases, the symmetry of the interactions is preserved and, consequently, isotropic adlayers are observed. However, most real components,

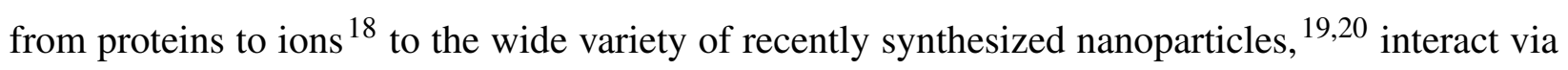
anisotropic or "patchy" attractions and clear signals of symmetry breaking have been observed. Experimental realization of such systems is growing. An example of real patchy particles is presented in Ref. 21 Such particles offer the possibility to be used as building blocks of specifically designed self-assembled structures. $19[20[22-24$

The term "molecular self-assembly" may be used to refer to spontaneous formation of an ordered molecular overlayer on a surface. Molecular self-assembly on surfaces via weak but selective noncovalent interactions, offers a promising bottom-up approach to fabricate highly organized systems from instructed molecular building blocks. In this way, the engineering of supramolecular arrays, with desired functionalities, on metal surfaces can be performed. ${ }^{25}$ If, in addition, such systems are capable of undergoing phase transitions, then their functional properties may be increased.

Recently, several research groups reported on the assembly of colloidal particles in linear chains. Selectively functionalizing the ends of hydrophilic nanorods with hydrophobic polymers, Nie et al. reported the observation of rings, bundles, chains, and bundled chains. $\frac{26}{}$ In another experimental study carried out by Chang et al., 27 gold nanorods were assembled into linear chains using a biomolecular recognition system.

Another prominent case is the self-assembly of flexible one-dimensional coordination polymers on metal surfaces, i.e. in Ref., ${ }^{28}$ where the researchers employed a de novo synthesized porphyrin module to construct one-dimensional (1D) $\mathrm{Cu}$-coordinated polymers on $\mathrm{Cu}(111)$ and $\operatorname{Ag}(111)$ surfaces. 
In a direct relation with the present work, Clair et al. ${ }^{29}$ investigated the self-assembly of terephthalic acid (TPA) molecules on the $\mathrm{Au}(111)$ surface. Using scanning tunneling microscopy, the authors showed that the TPA molecules arrange in one-dimensional chains with a discrete number of orientations relative to the substrate. However, in the experimental studies of chains selfassembled on a surface with a discrete number of orientations, orientational ordering transitions were not studied.

In this context, the main objective of this paper is to investigate the adsorption process in a system composed of monomers with two attractive (sticky) poles that self-assemble reversibly into polydisperse chains and, at the same time, undergo an orientational transition. In a recent series of papers,, $30-37$ the critical behavior of this system has been widely studied. Except mean-field calculations, $\frac{34}{}$ these studies showed the existence of a continuous phase transition along the entire coexistence curve. However, the universality class of the model has been a subject of controversy. ${ }^{36] 37}$ It was shown that the system under study represents an interesting case where the use of different statistical ensembles (canonical or grand canonical) leads to different and well-established universality classes ( $q=1$ Potts-type or $q=2$ Potts-type, respectively). $\stackrel{36}{ }$ The present work goes a step further addressing the study of the adsorption isotherms in different regions of the phase diagram, emphasizing their behavior at critical and subcritical temperatures. The adsorption isotherm appears as a sensitive quantity to the phase transition, allowing a very accurate determination of the phase diagram. These findings may be of interest for theoretical and experimental studies on critical adsorption phenomena.

It is clear that the model considered here is highly idealized and is not meant to reproduce a particular experimentally studied system. However, the understanding of simple models with increasing complexity might be a help and a guide for future experimental investigations. This work represents an effort in that direction.

The paper is organized as follows. In Sec. 2, lattice-gas model and theoretical formalism (mean-field approximation and exact functions for non-interacting and 1D systems) are presented. Section 3 is devoted to describe the Monte Carlo simulation scheme. The analysis of the results 
and discussion are given in Sec. 4. Finally, the conclusions are drawn in Sec. 5.

\section{Lattice-Gas Model and Theory}

As in Refs., $30-37$ a system of self-assembled rods with a discrete number of orientations in two dimensions is considered. The substrate is represented by a square or triangular lattice of $M=L \times L$ adsorption sites, with periodic boundary conditions. $N$ particles are adsorbed on the substrate with $m$ possible orientations along the principal axis of the array, being $m=2$ for square lattices and $m=3$ for triangular lattices (see Fig. 1). These particles interact with nearest-neighbors (NN) through anisotropic attractive interactions. Thus, a cluster or uninterrupted sequence of bonded particles is a self-assembled rod. Then, the grand canonical Hamiltonian of the system is given by

$$
H=w \sum_{\langle i, j\rangle}\left|\vec{r}_{i j} \cdot \vec{\sigma}_{i}\right|\left|\vec{r}_{j i} \cdot \vec{\sigma}_{j}\right| \operatorname{div} 1-\left(\mu-\varepsilon_{o}\right) \sum_{i}\left|\vec{\sigma}_{i}\right|
$$

where $\langle i, j\rangle$ indicates a sum over NN sites; $w$ represents the NN lateral interaction between two neighboring $i$ and $j$, here $w<0$ and the energy is lowered by an amount $|w|$ only if the NN monomers are aligned with each other and with the intermolecular vector $\vec{r}_{i j}$; $\vec{\sigma}_{j}$ is the occupation vector, with $\vec{\sigma}_{j}=0$ if the site $j$ is empty and $\vec{\sigma}_{j}=\hat{x}_{i}$ if the site $j$ is occupied by a particle with orientation along the $x_{i}$-axis; and $\varepsilon_{o}$ is the adsorption energy of an adparticle on a site. In the present work, $\varepsilon_{o}$ was set equal to zero (without any loss of generality) and the chemical potential $\mu$ is the only parameter that determines the strength of the adsorption. The operation div 1 , which is redundant in the case of square lattices, (i) avoids additional lateral interactions ${ }^{38}$ that promote the condensation of the monomers in the triangular lattice; and (ii) restricts the attractive couplings only to those pairs of NN monomers whose orientations are aligned with each other and with the monomer-monomer lattice direction, in line with the model on the square lattice. The sign of $\mu$ is chosen so that negative $\mu$ makes vacancies favorable. At $\mu=+\infty$, vacancies are suppressed and we wind up with the full lattice case. At $\mu=-\infty$, all we have are vacancies and the ground state is just vacant. 
The introduction of intermolecular forces brings about the possibility of phase transitions. $12[39[40$ Among the common types of phase transitions are, condensation of gases, melting of solids, transitions from paramagnet to ferromagnet and order-disorder transitions. From a theoretical point of view, when NN interactions are present, an extra term in the partition function for interaction energy is required. With this extra term, only partition functions for the whole system can be written. Ising ${ }^{41}$ gave an exact solution to the one-dimensional lattice problem in 1925. All other cases are

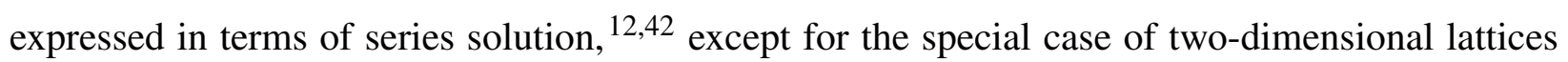
at half-coverage, which was exactly solved by Onsager ${ }^{43}$ in 1944 . Close approximate solutions in dimensions higher than one can be obtained, and the most important of these is the Bragg-Williams approximation (BWA). BWA is the simplest mean-field treatment for interacting adsorbed particles, even in the case of asymmetric particles as studied here. In this theoretical framework, the isotherm equation takes the form $12 \mid 44$

$$
K(c) e^{\left(\mu-\varepsilon_{o}-c w \theta\right) / k_{B} T}=\frac{\theta}{1-\theta}
$$

where $T$ is the temperature, $k_{B}$ is the Boltzmann constant, $\theta=N / M$ is the surface coverage and $K(c)$ represents the number of available configurations (per lattice site) for a monomer at zero coverage. The term $K(c)$ is, in general, a function of the connectivity of the lattice $c$ and the structure of the adsorbate ( $c=4$ and 6 for square and triangular lattices, respectively). It is easy demonstrate that $K(c)=2$ for square lattices and $K(c)=3$ for triangular lattices. $\frac{45}{4}$

In the limit of high temperatures $\left(w / k_{B} T \rightarrow 0\right)$, particles adsorb randomly on the surface and Eq. (??) reduces to the well-known Langmuir isotherm: 12

$$
K(c) e^{\left(\mu-\varepsilon_{o}\right) / k_{B} T}=\frac{\theta}{1-\theta} .
$$

On the other hand, the phase diagram reported in Ref. .34 indicates that, by decreasing temperature or increasing density, the monomers polymerize reversibly and form linear chains on the surface. In this limit, it is more convenient to describe the system from the exact form of the 
adsorption isotherm corresponding a interacting monomers on 1D lattices: $\frac{12}{12}$

$$
K(c) e^{\left(\mu-\varepsilon_{o}-w\right) / k_{B} T}=\frac{b-1+2 \theta}{b+1-2 \theta}
$$

where $b=\left\{1-4 \theta(1-\theta)\left[1-\exp \left(-w / k_{B} T\right)\right]\right\}^{1 / 2}$ and $K(c)=1$ for $1 \mathrm{D}$ lattices.

\section{Monte Carlo Simulation}

The thermodynamic properties of the present model were investigated following a standard importance sampling MC method in the grand canonical ensemble. ${ }^{46}$ The procedure is as follows. For a given pair of values of $T$ and $\mu$, an initial configuration with $N$ monomers adsorbed at random positions and orientations (on $M$ sites) is generated. Then an adsorption-desorption process is started, where the lattice sites are tested to change its occupancy state with probability given by the Metropolis rule: ${ }^{47} P=\min \{1, \exp (-\beta \Delta H)\}$, where $\Delta H$ is the difference between the Hamiltonians of the final and initial states and $\beta=1 / k_{B} T$. Insertion and removal of monomers, with a given orientation, are attempted with equal probability. For this purpose, an axis orientation (with probability $1 / 2$ for the square lattice, and $1 / 3$ for the triangular lattice) and a lattice site are chosen at random. If the selected lattice site is unoccupied, an attempt to place a monomer (with the orientation previously chosen) on the site is made. If, instead, the site is occupied, then the algorithm checks the orientational state of the adsorbed monomer and if this coincides with the previously chosen orientation, an attempt to desorb the particle is performed; otherwise, the trial ends. A Monte Carlo Step (MCS) is achieved when $M$ sites have been tested to change its occupancy state.

The equilibrium state can be well reproduced after discarding the first $s_{0}=10^{5}-10^{6} \mathrm{MCS}$. Then, averages are taken over $s=10^{5}-10^{6}$ successive configurations.

In this framework, total and partial adsorption isotherms can be obtained as simple averages:

$$
\theta=\frac{1}{M} \sum_{i}^{M}\left\langle\left|\vec{\sigma}_{i}\right|\right\rangle
$$


and

$$
\theta_{x_{i}}=\frac{1}{M} \sum_{i}^{M}\left\langle\left|\vec{\sigma}_{i} \cdot \hat{x}_{i}\right| \operatorname{div} 1\right\rangle,
$$

where $\theta$ represents the total surface coverage; $\theta_{x_{i}}$ denotes the partial surface coverage referring to the adparticles with orientation along the $x_{i}$-axis $\left(\theta=\sum_{i=1}^{c / 2} \theta_{x_{i}}\right)$ and $\langle\ldots\rangle$ means the time average over the Monte Carlo simulation runs.

All calculations were carried out using the parallel cluster BACO of Universidad Nacional de San Luis, Argentina. This facility, located at Instituto de Física Aplicada, Universidad Nacional de San Luis-CONICET, San Luis, Argentina, consists of 50 CPUs each with an Intel Core i7 processor running at $2.93 \mathrm{GHz}$ and $512 \mathrm{MB}$ of RAM per core.

\section{Results and Discussion}

In the present section, the main characteristics of the thermodynamic functions given in ??, will be analyzed in comparison with simulation results for a lattice-gas of asymmetric monomers on 2D lattices. The computational simulations have been developed for square and triangular $L \times L$ lattices, with $L=100$, and periodic boundary conditions. With this lattice size we verified that finite-size effects are negligible.

In order to understand the basic phenomenology, it is instructive to begin by discussing the behavior of the simulation adsorption isotherms for square lattices and different values of the magnitude of the lateral interactions [Fig. 2]. Curves from right to left correspond to $w / k_{B} T=0,-1,-2$, $-3,-4,-5,-6,-7,-8,-9$ and -10 , respectively. As it can be observed, isotherms shift to lower values of the chemical potential and their slope increases as the ratio $|w| / k_{B} T$ increases. This behavior is typical of attractive systems. However, a notable difference is observed with respect to the case of symmetric monomers, ${ }^{[12}$ where two nearest-neighbors particles interact with an interaction energy $w$. In fact, a clear discontinuity (jump) is observed in the adsorption isotherms corresponding to symmetric monomers for interactions above a critical value $w>w_{c}$ (in absolute values). The inset of Fig. 2 shows a scheme of the described process. The marked jump, which has been observed 
experimentally in numerous systems, is indicative of the existence of a first-order phase transition. In this situation, the only phase which one expects is a lattice-gas phase at low coverage, separated by a two-phase coexistence region from a "lattice-fluid" phase at higher coverage. 48

On the other hand, the surface coverage varies continuously with the chemical potential for all cases shown in Fig. 2, and there is no evidence of the existence of a first-order phase transition. In other words, the mechanism for which the asymmetric monomers polymerize reversibly into chains at low temperatures (high values of $|w| / k_{B} T$ ) is not associated with the existence of a first-order phase transition in the adlayer. This point will be discussed in the next.

In Ref., ${ }^{34}$ the phase diagram of the system was calculated by using Monte Carlo simulations on a square lattice. The results are reproduced in Fig. 3(a) to facilitate the reader with a selfcontained presentation. Circles joined by lines represent Monte Carlo simulation data obtained

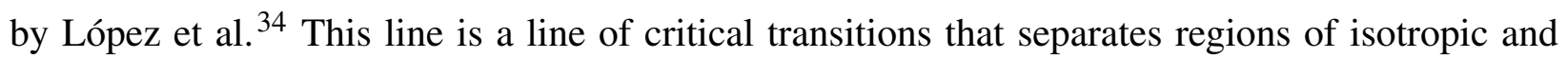
nematic stability.

In the following, we address the study of the adsorption isotherms in different regions of the phase diagram [different paths indicated in Fig. 3(a)], emphasizing their behavior at critical and subcritical temperatures.

Fig. 4(a) reports a comparison between theory (lines) and Monte Carlo simulation (symbols) for particles adsorbed on square (squares) and triangular (triangles) lattices. Two welldifferentiated limits have been considered in the figure: (i) non-interacting particles (full symbols): $w / k_{B} T=0$, path not shown in Fig. 3(a); and (ii) strongly interacting particles (open symbols): $w / k_{B} T=-8$, path (1) in Fig. 3(a), and $w / k_{B} T=-10$, path (2) in Fig. 3(a).

In the first case [(i)], lines correspond to results from ?? with $K(c)=2$ (square lattices) and $K(c)=3$ (triangular lattices). As expected, asymmetric monomers adsorb at random on the surface and an excellent agreement is obtained between theory and Monte Carlo simulation. The same behavior has been observed for values of $k_{B} T /|w|$ above $k_{B} T_{c}(\theta=1) /|w|$, being $T_{c}(\theta=1)$ the critical temperature characterizing the IN transition at full coverage. A typical configuration of the adlayer in this regime $\left[\theta=0.5\right.$ and $k_{B} T /|w|=0.65$, point $I$ in Fig. 3(a) $]$ is shown in Fig. 3(b). 
In the second case [(ii)], lines represent data from ??. From a first inspection of the curves it is observed that (a) the results corresponding to square and triangular lattices coincide in a unique curve; and (b) simulation data agree very well with theoretical results obtained for 1D lattices. These findings contribute to the understanding of the polymerization transition. Namely, in the case of strongly interacting particles (very low temperature limit), the IN phase transition occurs at very low coverage [see points $A$ and $B$ in Fig. 3(a)] and the asymmetric monomers adsorb forming chains over almost all range of coverage. A typical configuration of the adlayer in this regime $\left[\theta=0.6\right.$ and $k_{B} T /|w|=0.2$, point $J$ in Fig. 3(a)] is shown in Fig. 3(c).

Then, (1) the adsorption process behaves as a 1D problem, (2) the shape of the triangular and square isotherms are indistinguishable, and (3) at variance with the behavior observed for symmetric monomers (see inset of Fig. 2), the system studied here does not show a first-order phase transition at low temperature (it is well-known that no phase transition develops in a 1D lattice-gas $\frac{12}{12}$.

In Ref., ${ }^{34}$ the phase diagram corresponding to square lattices was also obtained by mean-field calculations. The results showed the existence of a coexistence region between a low-coverage isotropic phase and a high-coverage nematic one at low temperatures. The presence of this coexistence region (first-order phase transition) was completely at variance with the observed numerical simulation results. The study in Fig. 4(a) allows now to understand the mean-field prediction. Namely, the system goes to a 1D adsorption as the temperature decreases and, as it is well-known, mean-field approximation incorrectly predicts a phase transition in one dimension and low temperatures. $\frac{12}{12}$ The comparison in Fig. 4(b) confirms this argument. In the figure, simulation isotherms (symbols) obtained for square lattices and three different values of $w / k_{B} T$ (as indicated) are compared with the corresponding ones obtained from ?? (lines). The mean-field curves lead to the characteristic van der Waals loops and, consequently, to the prediction of a first-order phase transition. 12

Summarizing the discussion above, the adsorption process passes from a 2D problem (at high temperatures) to a $1 \mathrm{D}$ one (at very low temperatures). At the intermediate regime, the system 
behaves in a mixed regime and the simulation isotherms are not well fitted, neither by ?? nor by ?? (data not shown here). These findings provide very important information about the critical behavior of the system and, as it will be seen in the following, suggest a method to obtain the phase diagram of the system through adsorption measurements.

Let us suppose now that the system is found in the point $F$ of the phase diagram (intermediate temperature, isotropic region); with increasing coverage (at constant $k_{B} T /|w|$, in this case $k_{B} T /|w|=1 / 3$ ), the system passes (through the critical point $G$ ) from the non-oriented to the nematic phase at point $H$. The path $(F-G-H)$ is shown on the corresponding adsorption isotherm in Fig. 5(a). The figure also show a similar study for $k_{B} T /|w|=1 / 4$, path $(C-D-E)$ in Fig. 3(a). A detailed inspection of the curves in Fig. 5(a) reveals the existence of singularities in the critical points $D$ and $G$. To confirm this, the adsorption isotherms were differentiated with respect to the chemical potential. As an example, the results for the case $k_{B} T /|w|=1 / 3$ are shown in the inset of Fig. 5(b), where the critical coverage can be determined from the position of the maximum. The same methodology has been extended to other temperatures and the values obtained for the critical coverage (solid stars) agree very well with previous results from Ref. ${ }^{34}$

The existence of a singularity in the adsorption isotherms indicates that a dramatic change in the adsorption process takes place in the system. One way to visualize this situation is to consider the partial adsorption isotherms. As an example, total and partial adsorption isotherms are plotted in Fig. 5(b) for square lattices and $k_{B} T /|w|=1 / 3 . \quad \theta_{x_{1}}(\mu)$ and $\theta_{x_{2}}(\mu)$ represent the fraction of particles adsorbed along the $x_{1}$-axis (horizontal axis) and the fraction of particles adsorbed along the $x_{2}$-axis (vertical axis), respectively. Two well-differentiated adsorption regimes can be observed: (i) for $\theta<\theta_{c}$, particles are adsorbed isotropically on the surface and $\theta_{x_{1}}(\mu)=\theta_{x_{2}}(\mu)$, and (ii) for $\theta>\theta_{c}$, particles are adsorbed forming chains along one of the lattice axes (vertical axis in the case of the figure).

The procedure illustrated in Fig. 5 was used to obtain the temperature-coverage phase diagram corresponding to a triangular geometry. The results are shown in Fig. 6. Full circles represent data obtained from the inflection points in the adsorption isotherms. The figure also includes 
recent MC data by Almarza et al. $\frac{35}{35}$ (open circles). The rest of the curve separating isotropic and nematic stability (dashed line) was built on the basis of the previous results on the behavior of the system at low temperatures (see Fig. 4). Namely, the critical properties corresponding to square and triangular lattices coincide in the low-temperature (coverage) regime. The complete phase diagram of triangular lattices has been reported here for the first time. A more exact determination of $T_{c}$ based on Monte Carlo simulations and finite-size scaling theory is in progress.

For comparative purposes, Fig. 6 includes the critical line corresponding to square lattices (solid line). Even though the shapes of the curves are similar, the critical temperature corresponding to a given density $(\theta>0.1)$ is higher for square lattices than for triangular lattices.

In summary, the existence of singularities in the adsorption isotherms, which are induced by the phase transition, provides a way to determine the temperature-coverage phase diagram from adsorption experiments. These findings may be instructive for future theoretical and experimental studies on adsorption.

\section{Conclusions}

In the present paper, the main adsorption properties of self-assembled rigid rods on square and triangular lattices have been addressed. The results were obtained by using Monte Carlo simulations, mean-field theory and exact calculations in one dimension. According to the present analysis, the behavior of the system is characterized by the following properties:

1) At high temperatures, above the critical one characterizing the IN transition at full coverage $T_{c}(\theta=1)$, the particles are distributed isotropically on the surface, the adlayer behaves as a 2D system and an excellent agreement is observed between Monte Carlo simulation and theoretical results from Langmuir isotherm.

2) At very low temperatures, asymmetric monomers adsorb forming chains over almost all range of coverage and the adsorption process passes from a $2 \mathrm{D}$ problem to a $1 \mathrm{D}$ one. Then, 
the results corresponding to square and triangular lattices coincide in a unique curve, simulation data agree very well with exact theoretical results obtained for 1D lattices, and the system studied here does not show a first-order phase transition at low temperatures (it is well-known that no phase transition develops in a 1D lattice-gas ${ }^{12}$ ).

3) Mean-field calculations ${ }^{\sqrt{34}}$ showed the existence of a coexistence region (first-order phase transition) between a low-coverage isotropic phase and a high-coverage nematic one, at complete variance with the observed numerical simulation data. The result described in the item above allows now to understand the mean-field prediction. Namely, the system goes to a 1D adsorption as the temperature decreases and, as it is well-known, mean-field approximation incorrectly predicts a phase transition in one dimension. 12

4) At intermediate temperatures, the system exhibits a mixed behavior, the $2 \mathrm{D}$ and $1 \mathrm{D}$ adsorption processes are present in the adsorption isotherms and a marked singularity can be observed separating both regimes. The measurement of the point at which this singularity occurs (pronounced maximum in the derivative of $\theta$ with respect to $\mu$ ) allows an accurate determination of the critical coverage characterizing the IN phase transition.

5) The simple analysis described in the last item reproduces the previously obtained phase diagram for square lattices ${ }^{34}$ and provides a good approximation for the case of triangular lattices. A more detailed and accurate study, including the variation of critical exponents and the determination of the phase diagram through finite-size scaling analysis, is being undertaken for triangular lattices.

\section{Acknowledgement}

This work was supported in part by CONICET (Argentina) under project number PIP 112-20080101332; Universidad Nacional de San Luis (Argentina) under project 322000 and the National Agency of Scientific and Technological Promotion (Argentina) under project PICT-2010-1466. 


\section{Figure captions}

Fig. 1. Schematic representations of isotropic (left) and nematic (right) configurations of selfassembled rigid rods on square (top) and triangular (bottom) lattices. Here, the lattice size is $\mathrm{L}=30$.

Fig. 2. Simulation adsorption isotherms for square lattices and different values of the magnitude of the lateral interactions. Curves from right to left correspond to $w / k_{B} T=0,-1,-2,-3,-4,-5,-6,-7$, $-8,-9$ and -10 , respectively. Inset: Scheme showing the behavior of the adsorption isotherms for symmetric monomers and different values of $w / k_{B} T$ (above and below the critical value $w_{c} / k_{B} T$ ).

Fig. 3. (a) Phase diagram of the system on a square lattice: circles joined by lines represent Monte Carlo simulation data obtained by López et al. $\stackrel{34}{34}$ and stars correspond to results obtained from the singularities in the adsorption isotherms. The meaning of the dotted lines and the points $A-J$ is discussed in the text. (b) Simulation snapshot obtained in the isotropic region [point $I$ in Fig. 3(a)]. (c) Same as (b) for the nematic phase [point $J$ in Fig. 3(a)]. In both cases, the lattice size is $L=100$ and vertical and horizontal rods are shown in different colors for clarity.

Fig. 4. (a) Comparison between theoretical (lines) and simulation (symbols) isotherms for particles adsorbed on square $(c=4)$ and triangular $(c=6)$ lattices. Curves correspond to different values of $w / k_{B} T$ as indicated. In the case of $w / k_{B} T=0\left[w / k_{B} T=-8\right.$ and -10], lines correspond to results from ?? [??]. (b) Lattice coverage $\theta$ versus relative chemical potential $\mu / k_{B} T$ for square lattices and different values of $w / k_{B} T$ as indicated. Solid lines correspond to MFA [??] and symbols represent results from Monte Carlo simulation.

Fig. 5. (a) Simulation adsorption isotherms obtained for $w / k_{B} T=-3$ and $w / k_{B} T=-4$ as indicated. Points $C, D, E, F, G$ and $H$, corresponding to the phase diagram in Fig. 3(a), are indicated 
on the curves. (b) Total and partial adsorption isotherms obtained for $w / k_{B} T=-3$. Inset: Derivative of the total isotherm with respect to the chemical potential. The critical coverage can be determined from the position of the maximum.

Fig. 6. Phase diagram corresponding to asymmetric particles adsorbed on square (solid line) and triangular (dashed line) lattices. Full circles represent results obtained from the singularities in the adsorption isotherms and open circles correspond to MC data by Almarza et al. 35 


\section{References}

(1) Steele, W. A. The Interaction of Gases with Solid Surfaces; Pergamon Press: New York, 1974.

(2) Keller, J.; Staudt, R. Gas adsorption equilibria: experimental methods and adsorption isotherms; Springer: Boston, 2005.

(3) Tóth, J. Adsorption: Theory, Modeling, and Analysis; Dekker: New York, 2002.

(4) Dash, J. G. Films on Solid Surfaces; Academic Press: New York, 1975.

(5) Dash, J. G.; Ruvalds, J. Phase Transitions in adsorbed Films; Plenum: New York, 1980.

(6) Shina, S. K. Ordering in Two Dimensions; Elsevier: New York, 1980.

(7) Binder, K.; Landau, D. P. Multicritical Phenomena at Surfaces. Surf. Sci. 1976, 61, 577.

(8) Patrykiejew, A.; Sokolowski, S.; Binder, K. Phase Transitions in Adsorbed Layers Formed on Crystals of Square and Rectangular Surface Lattice. Surface Science Reports 2000, 37, 207.

(9) Rudziński, W.; Everett, D. H. Adsorption of Gases on Heterogeneous Surfaces; Academic Press: New York, 1992.

(10) Jaroniec, M. J.; Madey, R. Physical Adsorption on Heterogeneous Surfaces; Elsevier: Amsterdam, 1988.

(11) Rudziński, W.; Steele, W. A.; Zgrablich, G. Equilibria and Dynamics of Gas Adsorption on Heterogeneous Solid Surfaces ; Elsevier: Amsterdan, 1996.

(12) Hill, T. L. An Introduction to Statistical Thermodynamics; Addison Wesley Publishing Company: Reading, MA, 1960.

(13) Zhdanov, V. P. Elementary Physicochemical Processes on Solid Surfaces; Plenum Press: New York, 1991. 
(14) Tovbin, Y. K. Theory of Physical Chemistry Processes at a Gas-Solid Interface; Mir: Moscow, 1991.

(15) Ramirez-Pastor, A.J.; Bulnes, F.M.; Riccardo, J.L. Adsorption and transport of particles in low-dimensional heterogeneous substrates. Surf. Sci. 1999, 426, 48.

(16) Adamson, A. W. Physical Chemistry of Surfaces; Wiley: New York, 1990.

(17) Gregg, S. J.; Sing, K. S. W. Adsorption, Surface Area, and Porosity; Academic Press: New York, 1991.

(18) De Yoreo, J.; Vekilov, P. Principles of Crystal Nucleation and Growth. Rev. Mineral. Geochem. 2003, 54, 57.

(19) Glotzer, S. C. Some Assembly Required. Science 2004, 306, 419.

(20) Glotzer, S. C.; Solomon, M. J. Anisotropy of building blocks and their assembly into complex structures. Nature Mater. 2007, 6, 557.

(21) Cho, Y.-S.; Yi, G.-R.; Lim, J.-M.; Kim, S.-H.; Manoharan, V. N.; Pine, D. J.; Yang, S.M. Self-organization of bidisperse colloids in water droplets. J. Am. Chem. Soc. 2005, 127, 15968.

(22) Whitesides, G. M.; Boncheva, M. Beyond molecules: Self-assembly of mesoscopic and macroscopic components. Proc. Natl. Acad. Sci. U.S.A. 2002, 99, 4769.

(23) Zhang, Z.; Horsch, M. A.; Lamm, M. H.; Glotzer, S. C. Tethered Nano Building Blocks: Toward a Conceptual Framework for Nanoparticle Self-Assembly. Nano Lett. 2003, 3, 1341.

(24) Zhang, Z.; Glotzer, S. C. Self-Assembly of Patchy Particles. Nano Lett. 2004, 4, 1407.

(25) Barth, J. V. Molecular Architectonic on Metal Surfaces. Annu. Rev. Phys. Chem. 2007, 58, 375. 
(26) Nie, Z.; Fava, D.; Rubinstein, M.; Kumacheva, E. "Supramolecular" Assembly of Gold Nanorods End-Terminated with Polymer "Pom-Poms": Effect of Pom-Pom Structure on the Association Modes. J. Am. Chem. Soc. 2008, 130, 3683.

(27) Chang, J. Y.; Wu, H.; Chen, H.; Ling, Y. C.; Tan, W. Oriented Assembly of Au Nanorods Using Biorecognition System. Chem. Commun. 2005, 8, 1092.

(28) Heim, D.; Écija, D.; Seufert, K.; Auwärter, W.; Aurisicchio, C.; Fabbro, C.; Bonifazi, D.; Barth, J. V. Self-Assembly of Flexible One-Dimensional Coordination Polymers on Metal Surfaces. J. Am. Chem. Soc. 2010, 132, 6783.

(29) Clair, S.; Pons, S.; Seitsonen, A. P.; Brune, H.; Kern, K.; Barth, J. V. STM Study of Terephthalic Acid Self-Assembly on Au(111): Hydrogen-Bonded Sheets on an Inhomogeneous Substrate. J. Phys. Chem. B 2004, 108, 14585.

(30) Tavares, J. M.; Holder, B.; Telo Da Gama, M. M. Structure and phase diagram of selfassembled rigid rods: equilibrium polydispersity and nematic ordering in two dimensions. Phys. Rev. E 2009, 79, 021505.

(31) López, L. G.; Linares, D. H.; Ramirez-Pastor, A. J. Critical exponents and universality for the isotropic-nematic phase transition in a system of self-assembled rigid rods on a lattice. Phys. Rev. E 2009, 80, 040105.

(32) Almarza, N. G.; Tavares, J. M.; Telo Da Gama, M. M. Effect of polydispersity on the ordering transition of adsorbed self-assembled rigid rods. Phys. Rev. E 2010, 82, 061117.

(33) López, L. G.; Linares, D. H.; Ramirez-Pastor, A. J. Critical behavior of self-assembled rigid rods on triangular and honeycomb lattices. J. Chem. Phys. 2010, 133, 134702.

(34) López, L. G.; Linares, D. H.; Ramirez-Pastor, A. J.; Cannas, S. A. Phase diagram of selfassembled rigid rods on two-dimensional lattices: Theory and Monte Carlo simulations. $J$. Chem. Phys. 2010, 133, 134706. 
(35) Almarza, N. G.; Tavares, J. M.; Da Gama, M. M. T. Communication: The criticality of selfassembled rigid rods on triangular lattices. J. Chem. Phys. 2011, 134, 071101.

(36) López, L. G.; Linares, D. H.; Ramirez-Pastor, A. J. Comment on "Effect of polydispersity on the ordering transition of adsorbed self-assembled rigid rods." Phys. Rev. E 2012, 85, 053101.

(37) Almarza, N. G.; Tavares, J. M.; Telo Da Gama, M. M. Reply to "Comment on 'Effect of polydispersity on the ordering transition of adsorbed self-assembled rigid rods'." Phys. Rev. E 2012, 85, 053102.

(38) If the operation div 1 is not used in ??, there will be three possible values of energy between each pair of nearest-neighbor monomers on the triangular lattice: $w$ if they are aligned in the direction of the intermolecular vector $\vec{r}_{i j} ; w / 2$ if one is aligned in the direction of $\vec{r}_{i j}$ and the other not; and $w / 4$ if they are oriented in one direction but different to $\vec{r}_{i j}$.

(39) Goldenfeld, N. Lectures on Phase Transitions and the Renormalization Group; Addison Wesley: Reading, MA, 1992.

(40) Yeomans, J. M. Statistical Mechanics of Phase Transitions; Clarendon Press: Oxford, 1992.

(41) Ising, E. Beitrag zur Theorie des Ferromagnetismus. Z. Physik 1925, 31, 253.

(42) (a) Domb, C. In Phase transition and critical phenomena; Domb, C., Green, M. S., Eds.; Academic Press: London, New York, 1974; Vol. 3, p 1. (b) Fisher, M. E. The theory of equilibrium critical phenomena. Rep. Prog. Phys. 1967, 30, 615.

(43) Onsager, L. Crystal Statistics. I. A Two-Dimensional Model with an Order-Disorder Transition. Phys. Rev. 1944, 65, 117.

(44) Ramirez-Pastor, A. J.; Aligia, A.; Romá, F.; Riccardo, J. L. Multisite-Occupancy Adsorption and Surface Diffusion of Linear Adsorbates in Low Dimensions: Rigorous Results for a Lattice Gas Model. Langmuir 2000, 16, 5100. 
(45) Romá, F.; Riccardo, J. L.; Ramirez-Pastor, A. J. Application of the Fractional Statistical Theory of Adsorption (FSTA) to Adsorption of Linear and Flexible $k$-mers on Two-Dimensional Surfaces. Ind. Eng. Chem. Res. 2006, 45, 2046.

(46) Binder, K. Applications of the Monte Carlo Method in Statistical Physics. Topics in current Physics; Springer: Berlin, 1984; Vol. 36.

(47) Metropolis, N.; Rosenbluth, A.; Rosenbluth, M.; Teller, A.; Teller, E. Equation-of-State Calculations by Fast Computing Machines. J. Chem. Phys. 1953, 21, 1087.

(48) In the case of square lattices and symmetric particles, this phase transition occurs for $w / k_{B} T<w_{c} / k_{B} T=2 \ln (\sqrt{2}-1) .49$

(49) Kramers, H. A.; Wannier, G. H. Statistics of the Two-Dimensional Ferromagnet. Part I. Phys. Rev. 1941, 60, 252. 

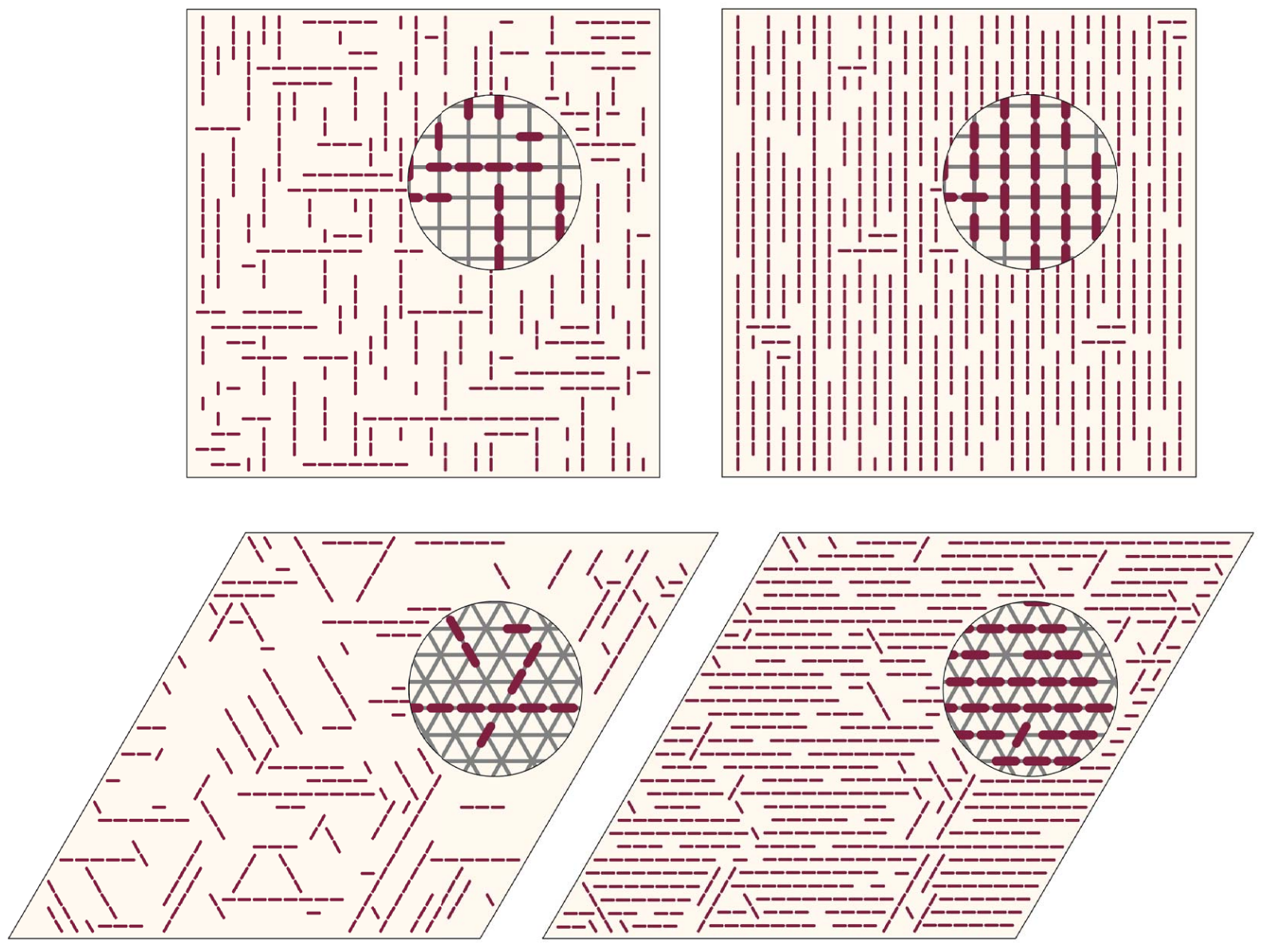

Figure 1 


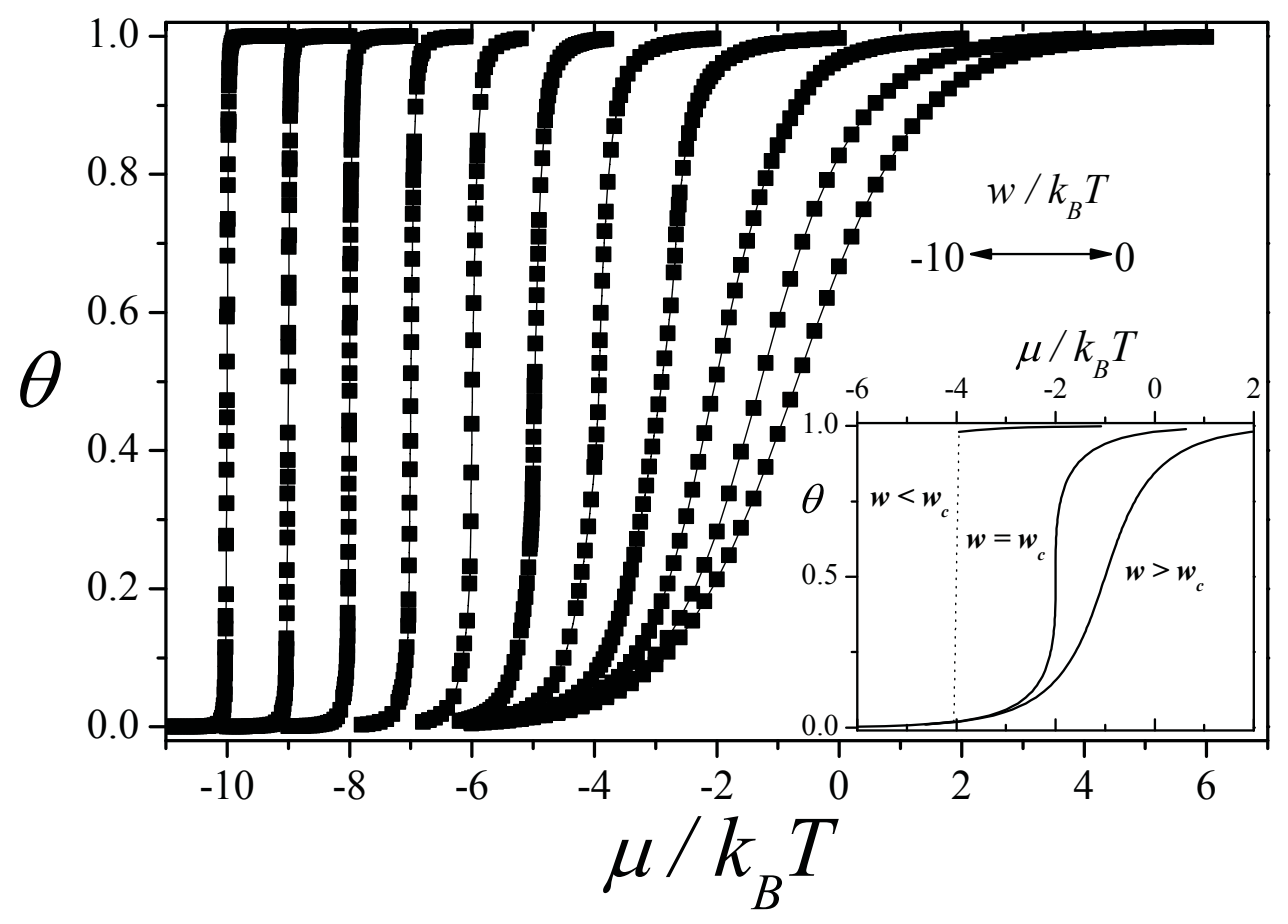

Figure 2 


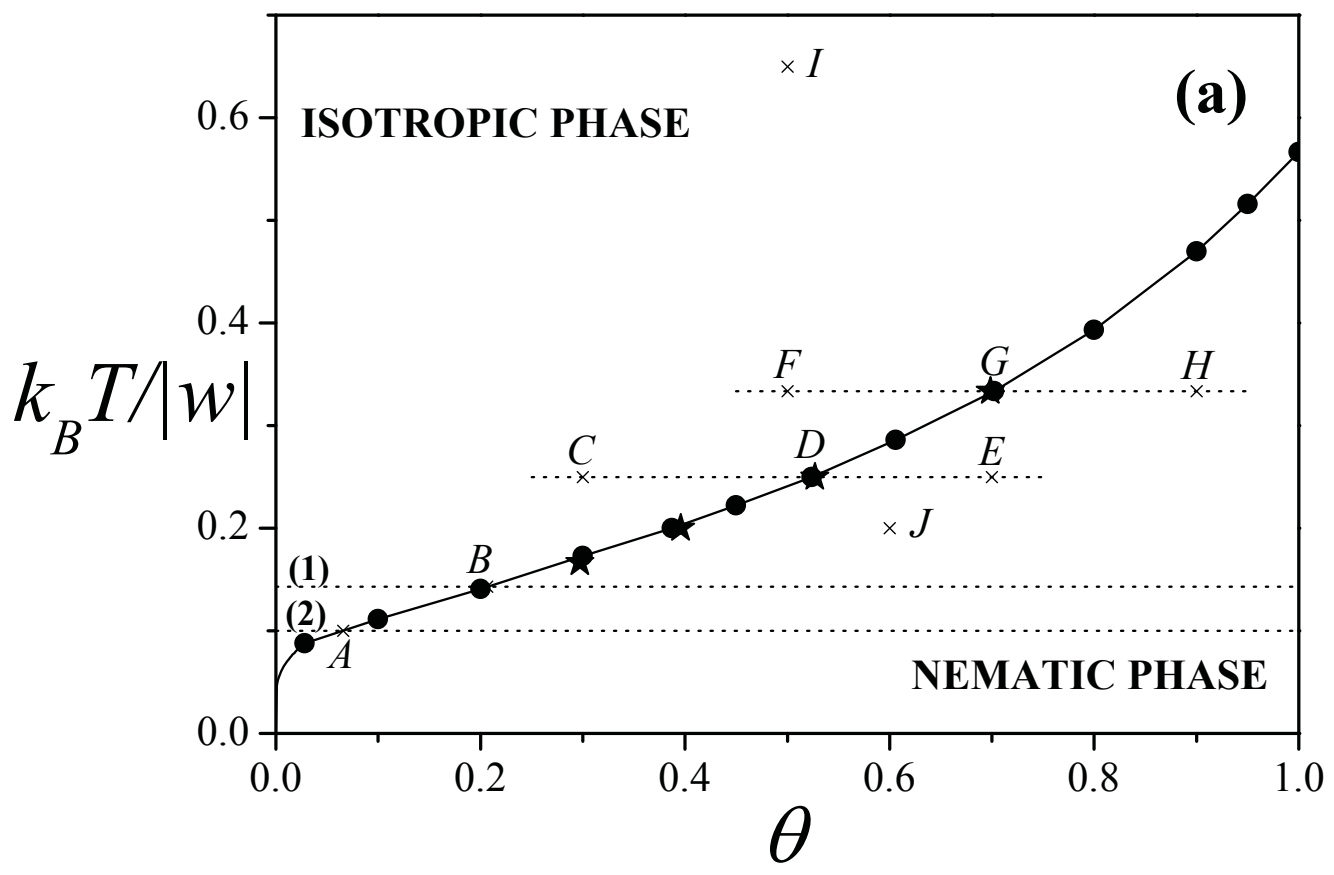

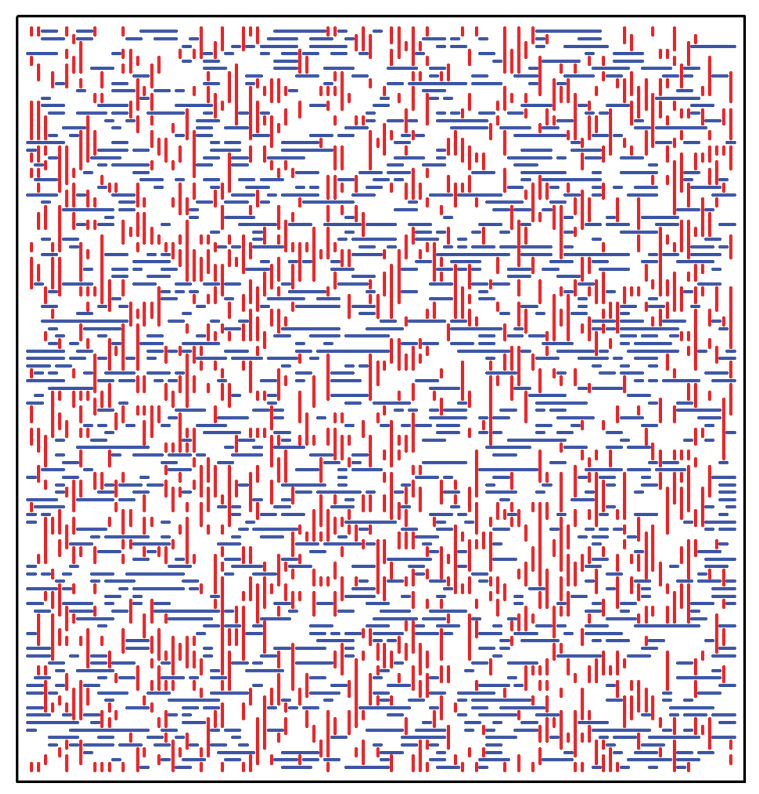

(b)

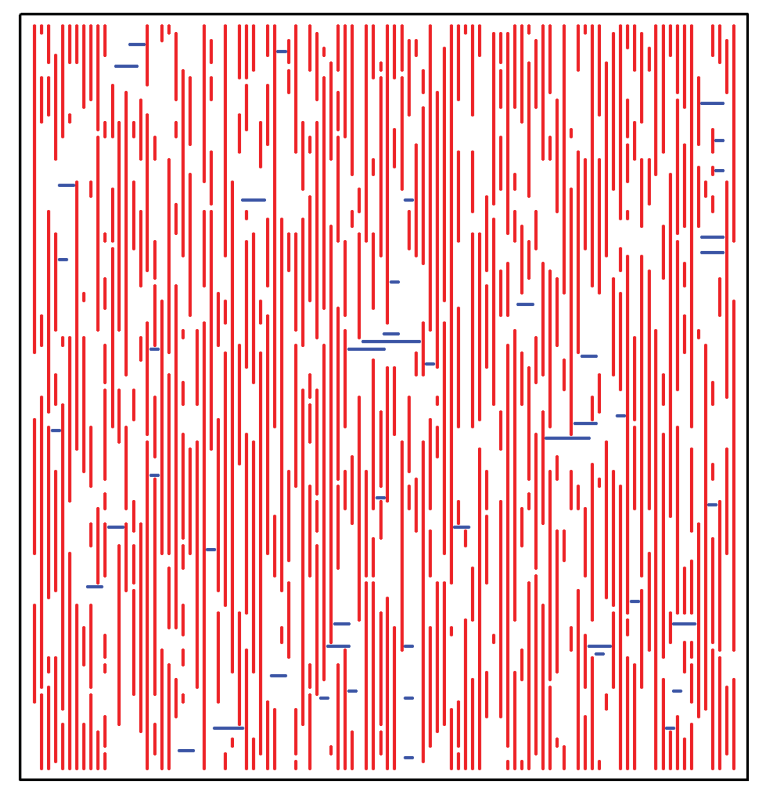

(c)

Figure 3 

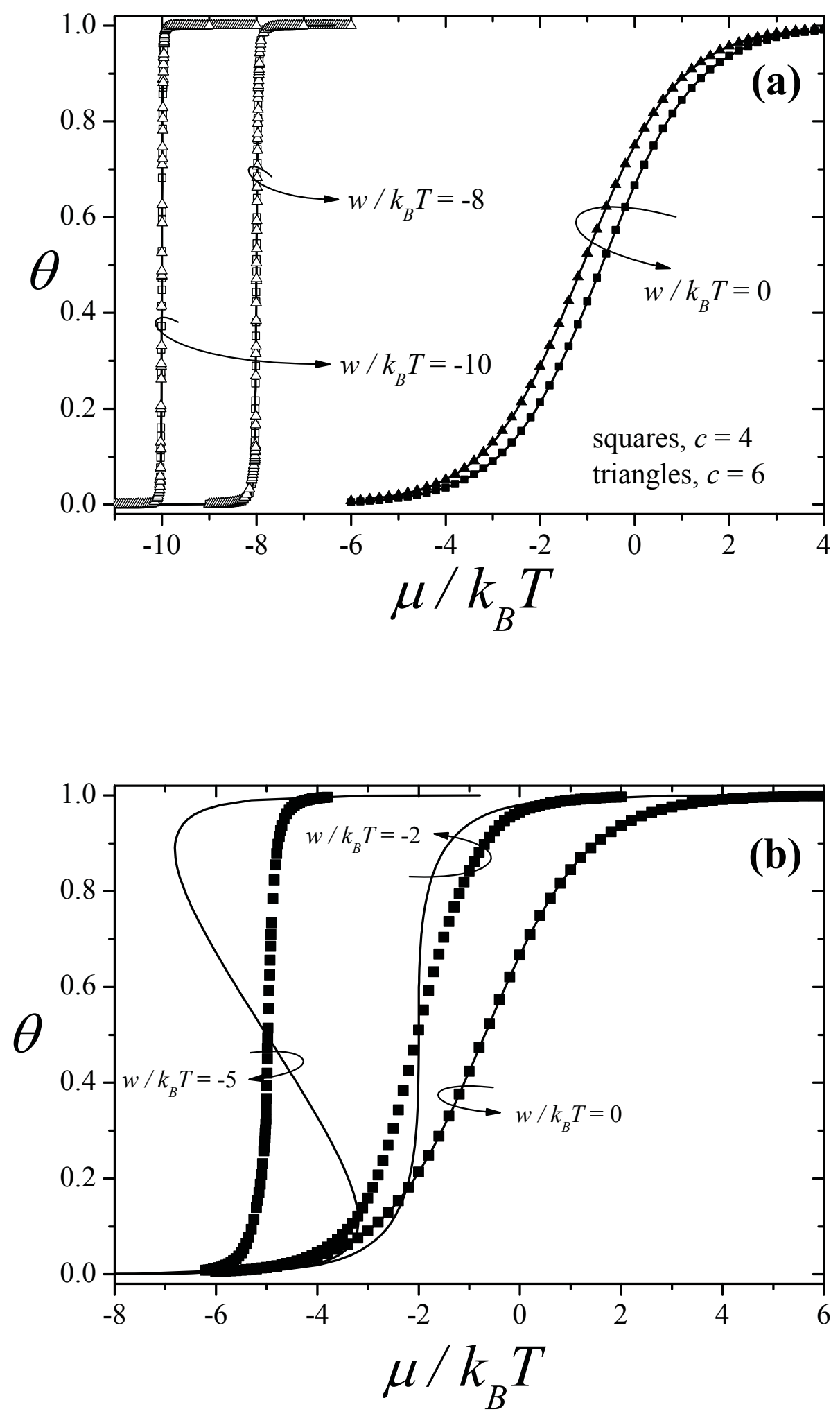

Figure 4 

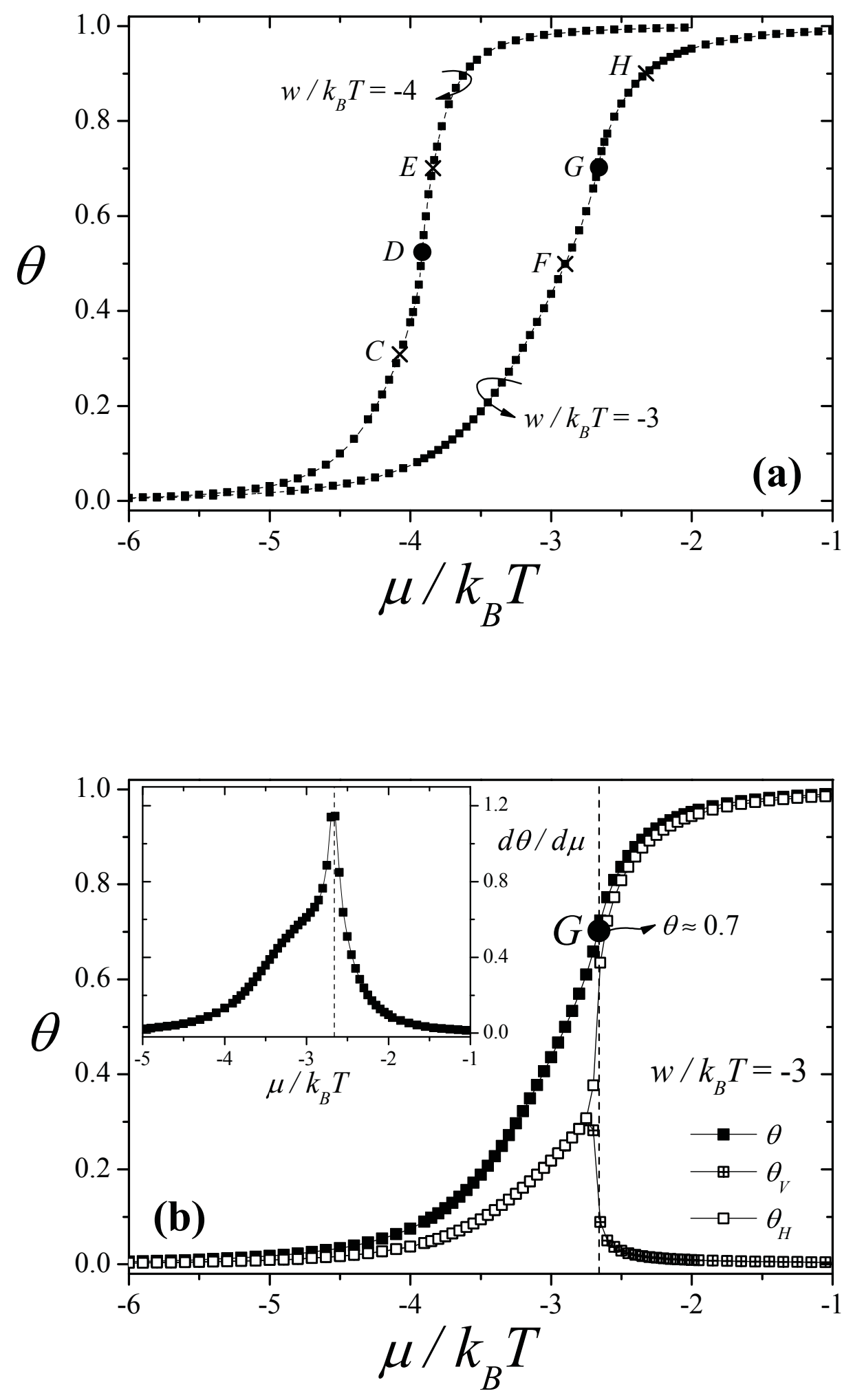

Figure 5 


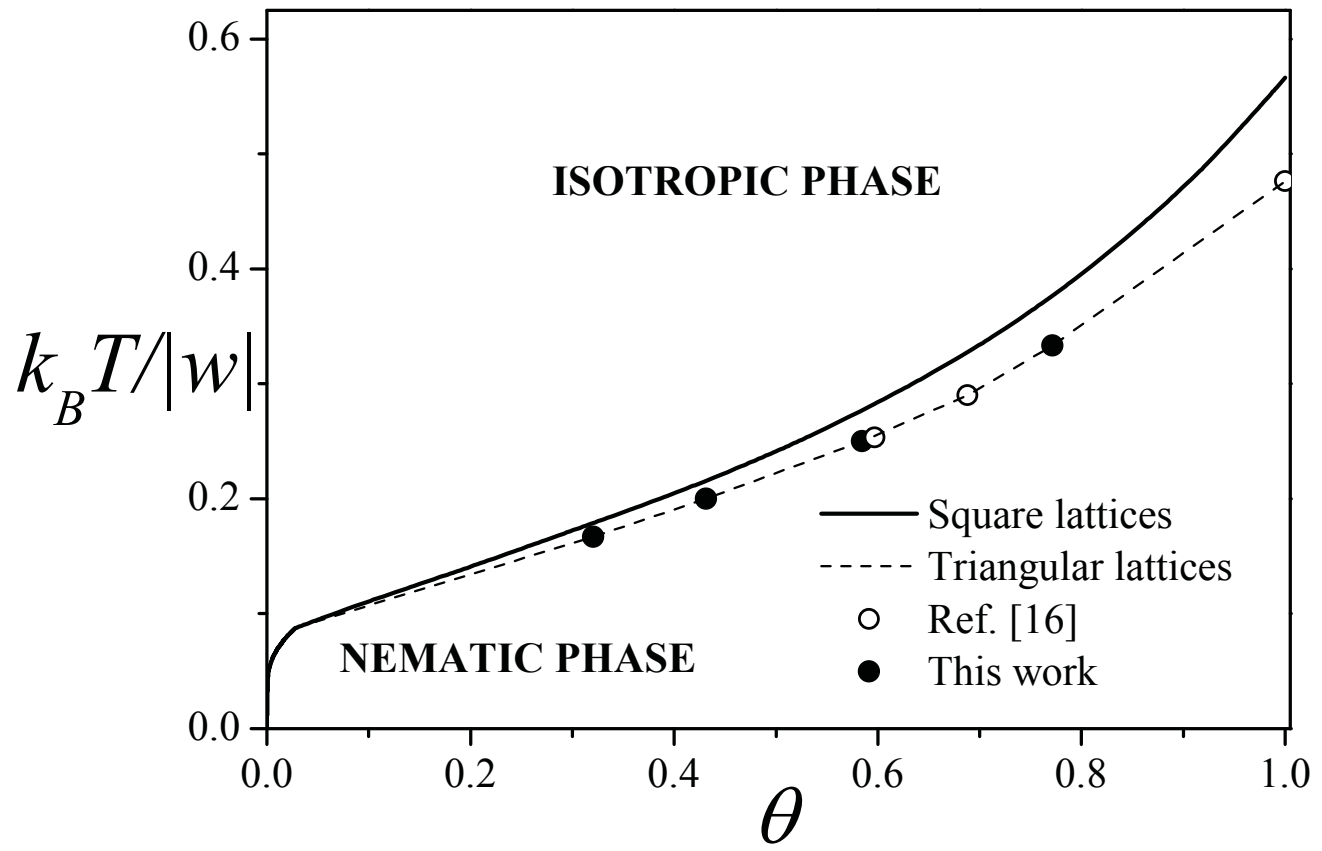

Figure 6 